\title{
Muscle Weakness Right-Sided
}

National Cancer Institute

\section{Source}

National Cancer Institute. Muscle Weakness Right-Sided. NCI Thesaurus. Code C78457.

A reduction in the strength of the muscles in the right side of the body. 